\title{
GATA4-dependent regulation of the secretory phenotype via MCP-1 underlies lamin A-mediated human mesenchymal stem cell aging
}

\author{
Jin Young Lee ${ }^{1,2}$, Kyung-Rok Yu ${ }^{1,2,3}$, Byung-Chul Lee ${ }^{1,2}$, Insung Kang ${ }^{1,2}$, Jae-Jun Kim,2, Eui-Jung Jung ${ }^{1,2}$, \\ Hyung-Sik Kim ${ }^{4,5}$, Yoojin Seo ${ }^{4,5}$, Soon Won Choi $\mathbb{B}^{1,2}$ and Kyung-Sun Kang ${ }^{1,2}$
}

\begin{abstract}
Defects in the nuclear lamina occur during physiological aging and as a result of premature aging disorders. Aging is also accompanied by an increase in transcription of genes encoding cytokines and chemokines, a phenomenon known as the senescence-associated secretory phenotype (SASP). Progerin and prelamin A trigger premature senescence and loss of function of human mesenchymal stem cells (hMSCs), but little is known about how defects in nuclear lamin A regulate SASP. Here, we show that both progerin overexpression and ZMPSTE24 depletion induce paracrine senescence, especially through the expression of monocyte chemoattractant protein-1 (MCP-1), in hMSCs. Importantly, we identified that GATA4 is a mediator regulating MCP-1 expression in response to prelamin A or progerin in hMSCs. Co-immunoprecipitation revealed that GATA4 expression is maintained due to impaired p62mediated degradation in progerin-expressing hMSCs. Furthermore, depletion of GATA4 abrogated SASP-dependent senescence through suppression of NF-KB and MCP-1 in hMSCs with progerin or prelamin A. Thus, our findings indicate that abnormal lamin A proteins trigger paracrine senescence through a GATA4-dependent pathway in hMSCs. This molecular link between defective lamin A and GATA4 can provide insights into physiological aging and pathological aging disorders.
\end{abstract}

\section{Introduction}

The LMNA gene encodes lamin A and lamin C, which are major components of the nuclear lamina. Mutations in the LMNA gene have been implicated in premature aging disorders, including Hutchinson-Gilford progeria syndrome (HGPS) ${ }^{1}$. HGPS is caused by a splicing defect and consequent generation of progerin, a mutanttruncated lamin A protein ${ }^{2}$. Cells of HGPS patients exhibit an abnormal nuclear structure, increased DNA

\footnotetext{
Correspondence: Kyung-Sun Kang (kangpub@snu.ac.kr)

${ }^{1}$ Adult Stem Cell Research Center, College of Veterinary Medicine, Seoul

National University, Seoul 08826, Republic of Korea

${ }^{2}$ College of Veterinary Medicine and Research Institute for Veterinary Science,

Seoul National University, Seoul 08826, Republic of Korea

Full list of author information is available at the end of the article
}

damage and premature senescence ${ }^{3,4}$. In addition to the effects of progerin, accumulation of prelamin A, a precursor of lamin A, induces defects in nuclear structures. ZMPSTE24 is an enzyme that produces mature lamin A by cleavage of amino acids in prelamin A. Zmpste24 knock-out mice have been widely used to study the mechanisms of aging and progeria ${ }^{5}$. Depletion of Zmpste24 causes premature senescence in mice, including decreases in life span and bone density. Increased prelamin A expression caused by ZMPSTE24 deficiency causes defective DNA repair ${ }^{4,6}$. Zmpste24 knock-out mice have been extensively studied because of their impaired DNA damage response (DDR) ${ }^{7,8}$. Lamin A also functions as a structural barrier to $\mathrm{DDR}^{9,10}$. Altogether, these findings indicate that defects in the nuclear structure 
induced by progerin or prelamin A lead to the accumulation of DNA damage, which results in accelerated aging.

Scaffidi et al. reported that exogenous expression of progerin in hMSCs can impair their differentiation potential $^{11}$. Furthermore, production of induced pluripotent stem cells (iPSCs) from HGPS patients has revealed that the progerin expression levels are the highest in MSCs, vascular smooth muscle cells, and fibroblasts ${ }^{12}$. HGPS-iPSC-derived hMSCs display increased DNA damage and impaired therapeutic efficacy in murine ischemic hind limb models. These results indicate that MSCs are a specific target cell type of progerin-induced senescence. Like progerin, excessive accumulation of prelamin A induces premature senescence in MSCs, including wrinkled nuclei ${ }^{13,14}$. Downregulation of ZMPSTE24 in hMSCs also induces a senescence phenotype, including increased $\beta$ galactosidase $\left(\beta\right.$-gal) activity and DDR ${ }^{14}$. These investigations imply that both progerin and prelamin $\mathrm{A}$ can induce senescence in hMSCs with a change in nuclear morphology.

Senescent cells secrete a group of factors that induce senescence in neighboring cells, a phenomenon termed senescence-associated secretory phenotype $(\mathrm{SASP})^{15-18}$. The SASP is activated by the NF-kB and C/EBP $\beta$ pathways and involves several cytokines and chemokines ${ }^{19}$. Previous studies investigating SASP have demonstrated that oncogene-induced senescence (OIS) and DNA damage induce the secretion of senescence-associated inflammatory cytokines ${ }^{18,20-22}$. The secreted inflammatory factors propagate senescence and recruit immune cells to senescent tissues by the generation of a proinflammatory environment. Among the factors reported to regulate the SASP, GATA4 has been recently identified as a regulator of senescence and inflammation ${ }^{23,24}$. GATA4 is expressed during oncogene- and irradiationinduced senescence in fibroblasts in response to DNA damage. During the process of cellular senescence, GATA4 has a regulatory role in the SASP of fibroblasts through the NF- $\mathrm{kB}$ pathway. Because GATA4-dependent cellular senescence is closely associated with DDR, the role of GATA4 in other senescence models and other cell types may reveal a new mechanism.

Senescent hMSCs also induce senescence in neighboring cells. Monocyte chemoattractant protein-1 (MCP-1) secreted from senescent human umbilical cord bloodderived mesenchymal stem cells (hUCB-MSCs) induces premature senescence in neighboring cells ${ }^{25}$. Insulin-like growth factor binding proteins 4 and 7 are also produced by senescent hMSCs, and they trigger senescence in adjacent normal cells ${ }^{26}$. These studies investigated the mechanisms of the SASP by inducing senescence in hMSCs through prolonged passaging. However, cellular senescence of MSCs can be regulated by various factors other than passaging. In our previous report, we have demonstrated that depletion of ZMPSTE24 and introduction of progerin induce premature senescence in hUCB-MSCs ${ }^{14}$. It remains to be determined whether defective lamin A triggers paracrine senescence via inflammatory factors in hMSCs.

In this study, we identified that paracrine senescence is triggered in senescent hMSCs with abnormal nuclear structures by increasing the expression of MCP-1 and that inhibition of MCP-1 decreases the SASP. Furthermore, we found that GATA4 mediates the senescence of hMSCs induced by defective lamin A. We assessed whether down-regulation of GATA4 disturbs the progerin- or prelamin A-dependent senescence phenotype. Elucidating how GATA4 regulates senescence in hMSCs with nuclear defects may aid in understanding the etiology of complex aging disorders. We show that inhibition of GATA4 expression protects hMSCs from cellular senescence, implying a unique therapeutic opportunity against progeroid syndromes and physiological aging.

\section{Materials and methods \\ Isolation and culture of hUCB-MSCs}

For the isolation of hUCB-MSCs, UCB samples were obtained from umbilical veins immediately after delivery with informed consent from the mothers. The procedures were approved by the Boramae Hospital Institutional Review Board (IRB) and the Seoul National University IRB (IRB no. 1608/001-021). The hUCB-MSCs were isolated and cultured as previously described ${ }^{27}$. The isolated cells were grown in KSB-3 Complete Medium (Kangstem Biotech, Seoul, Korea) with $10 \%$ fetal bovine serum. We used hMSCs that had been verified to have characteristics of stem cells, as determined by their differentiation, proliferation, and surface marker expression.

\section{In vitro differentiation experiments}

In vitro differentiation of hUCB-MSCs was performed as previously described ${ }^{28}$.

\section{Isolation and culture of hUCB-derived mononuclear cells}

Human mononuclear cells were isolated from UCB samples as previously described ${ }^{29}$.

\section{Senescence-associated beta-galactosidase (SA- $\beta$-gal) staining}

SA- $\beta$-gal staining was conducted as previously described $^{14}$. Briefly, cultured hMSCs were washed with PBS and fixed with $0.5 \%$ glutaraldehyde in PBS ( $\mathrm{pH} 7.2$ ) for 5 min at room temperature. The cells were then washed with PBS containing $\mathrm{MgCl}_{2}(\mathrm{pH} 7.2,1 \mathrm{mM} \mathrm{MgCl} 2$ ) and stained with X-gal solution [1 mg/ml X-gal, $0.12 \mathrm{mM} \mathrm{K}_{3} \mathrm{Fe}(\mathrm{CN})_{6}$, $1 \mathrm{mM} \mathrm{MgCl} 2$ in PBS, pH 6.0] overnight at $37^{\circ} \mathrm{C}$. The cells 
were washed with PBS, and images were obtained using a microscope (IX70, Olympus, Tokyo, Japan).

\section{MTT assay}

The proliferative ability of hUCB-MSCs was measured using the 3-(4,5-dimethylthiazol-2-yl)-2,5-diphenyltetrazolium bromide (MTT) (Sigma-Aldrich, St. Louis, USA) assay as previously described ${ }^{28}$.

\section{Western blot analysis}

Western blot analysis was performed as previously described $^{28}$. The primary antibodies used to probe each protein were as follows: rabbit anti-p16 ${ }^{\mathrm{INK} 4 \mathrm{a}}$ (1:1000; Abcam, Cambridge, UK; ab108349), mouse anti-actin (1:3000, Santa Cruz, Texas, USA; sc-47778), mouse antiGATA4 (1:200, Santa Cruz; sc-25310), rabbit anti-p-p65 (1:1000, Cell Signaling, Massachusetts, USA; \#3033), goat anti-lamin A (1:250, Santa Cruz; sc-6214, sc-6215), and mouse anti-p62 (1:1000, BD, New Jersey, USA; 610832). Secondary horseradish peroxidase (HRP)-conjugated antibodies (1:2000; Invitrogen, Carlsbad, USA; G21040, G21234) were used according to the manufacturer's instructions, and binding was detected using an enhanced chemiluminescence (ECL) detection kit (Amersham Pharmacia Biotek, Amersham, UK).

\section{Conditioned medium}

Conditioned medium (CM) was generated by exposing cells to RPMI 1640 containing $1 \mathrm{mM}$ sodium pyruvate, 2 $\mathrm{mM}$ glutamine, minimum essential medium (MEM) vitamins, and MEM non-essential amino acids. hMSCs were washed three times with PBS and cultured with CM for $24 \mathrm{~h}$. After incubation, the CM was collected and centrifuged at $5000 \times g$, and the supernatants were collected.

\section{Mononuclear cell migration assay}

Migration assays were performed using Transwell polycarbonate membrane inserts with an $8-\mu \mathrm{m}$ pore diameter (SPL, Pocheon, South Korea). CM $(400 \mu \mathrm{l})$ collected from cells were introduced into the lower chambers of the plates in triplicate. Mononuclear cells $(\mathrm{MNCs})\left(3 \times 10^{5}\right)$ resuspended in RPMI-10 were plated onto the inserts. MNCs were allowed to migrate to the lower chamber for $6 \mathrm{~h}$ at $37^{\circ} \mathrm{C}$. At $6 \mathrm{~h}$, the number of cells in the lower chamber was quantified using a Cell Counting Kit-8 (Dojindo, Kumamoto, Japan) according to the manufacturer's instructions.

\section{Viral transduction}

The retroviral plasmid vectors, including pBABEpuro-control, pBABE-puro-GFP-wt-LMNA, pBABEpuro-GFP-progerin, shCTL-mLPx and mLPxshZMPSTE24, were purchased from Addgene. The
pInducer20-GATA4 and MSCV-GATA4 shRNA were provided by Dr. Stephen J. Elledge of Harvard medical school $^{24}$. Virus production and transduction were performed as previously described ${ }^{30}$. Briefly, the retroviral plasmids pBABE-puro-control, pBABE-puro-GFP-wtLMNA, pBABE-puro-GFP-progerin, shCTL-mLPx, mLPx-shZMPSTE24, and MSCV-GATA4 shRNA were transfected into 293FT cells with VSV-G and gag/pol plasmids using Fugene 6 transfection reagent (Roche, Basel, Switzerland). The viral supernatants were collected 48 and $72 \mathrm{~h}$ after transfection and were used to infect hUCB-MSCs in the presence of $5 \mu \mathrm{g} / \mathrm{ml}$ polybrene (Sigma). After the viral transduction, the hMSCs were selected with puromycin $(0.5 \mu \mathrm{g} / \mathrm{ml})$ for 4 days and cultured for 3 days before the analysis. The lentiviruses were generated according to the manufacturer (Thermo Fisher scientific, MA, USA) to introduce Dox-inducible flagGATA4. The viral supernatants were collected 48 and 72 $\mathrm{h}$ after transfection and were used to infect hUCB-MSCs $(\mathrm{MOI}=2)$ in the presence of $5 \mu \mathrm{g} / \mathrm{ml}$ polybrene (Sigma). At $24 \mathrm{~h}$ after transduction, the cells were washed four times in PBS and maintained in growth medium.

\section{Quantitative RT-PCR}

Total RNA was extracted using TRIzol (Invitrogen) according to the manufacturer's suggestion. The acquired RNA was reverse-transcribed to cDNA using the Superscript First-Strand Synthesis System (Invitrogen). Relative mRNA expression levels were determined using the SYBR Green PCR Master Mix (Applied Biosystems, Foster City, USA) with an ABI 7300 detection system and the supplied software. The expression level of each gene was normalized to GAPDH. At least three independent analyses were carried out for each gene.

\section{Immunocytochemistry}

Cells were fixed in $4 \%$ paraformaldehyde for $15 \mathrm{~min}$ at room temperature, permeabilized with $0.25 \%$ Triton X100 in PBS for $10 \mathrm{~min}$ and then blocked for $1 \mathrm{~h}$ with $5 \%$ normal goat serum (Zymed, San Francisco, USA) at room temperature. The cells were then stained with antibodies against indicated proteins, followed by incubation for $1 \mathrm{~h}$ at room temperature with Alexa-Fluor-488- or AlexaFluor-594-labeled secondary antibodies (1:1000; Molecular Probes, Oregon, USA). The nuclei were stained with Hoechst $33258(1 \mu \mathrm{g} / \mathrm{ml}$ for $5 \mathrm{~min})$, and images were captured using a confocal microscope (Eclipse TE200, Nikon, Japan).

\section{Co-immunoprecipitation}

Adherent cells $\left(2 \times 10^{7}\right)$ were washed two times with PBS and lysed in non-denaturing lysis buffer (1\% Triton $\mathrm{X}-100,50 \mathrm{mM}$ Tris, $\mathrm{pH}$ 7.4, $300 \mathrm{mM} \mathrm{NaCl}, 5 \mathrm{mM}$ EDTA and $0.02 \%$ sodium azide) with protease inhibitor 
(GenDEPOT, Texas, USA). Lysates were scraped from plates and then incubated overnight at $4{ }^{\circ} \mathrm{C}$ with the indicated antibody. Protein $\mathrm{A} / \mathrm{G}$ agarose beads were added to the antibody-lysate complex, and the mixture was incubated for an additional $4 \mathrm{~h}$ at $4{ }^{\circ} \mathrm{C}$. After incubation, the beads were washed three times with lysis buffer and resuspended in SDS loading buffer, and the protein complexes were eluted by boiling. The eluted samples were separated by $10-12 \%$ SDS-PAGE. The proteins were transferred to a nitrocellulose membrane and probed overnight at $4{ }^{\circ} \mathrm{C}$ with primary antibody followed by an appropriate secondary antibodies conjugated to horseradish peroxidase for $1 \mathrm{~h}$ at room temperature. The membrane was washed with TBST (0.05\% Tween 20/ Tris-buffered saline) and detected with ECL Plus Western blotting detection reagents.

\section{Statistical analysis}

Data are presented as representative examples or means of more than three experiments. Data are presented as the means \pm s.d. Two-tailed Student's $t$-test was used for statistical analysis throughout our experiments.

\section{Results}

\section{Progerin induces senescence phenotypes in hUCB-MSCs}

To determine whether progerin induces senescence in hUCB-MSCs, we used retroviruses to express GFPprogerin. Immunoblots confirmed the over-expression of GFP-progerin and increased expression of p16 ${ }^{\mathrm{INK}}$, a marker of senescence in hMSCs, as we previously reported (Fig. 1a) ${ }^{14}$. Induction of cellular senescence was also confirmed by the increase in SA- $\beta$-gal activity (Fig. 1b). MTT activity was decreased in response to progerin overexpression, indicating decreased proliferative activity (Fig. 1c). To assess whether progerin-expressing hMSCs can transmit the senescent phenotype in a paracrine manner, we co-cultured normal hMSCs and progerinoverexpressing hMSCs in transwell chambers. The SA- $\beta$ gal activity was significantly increased when hMSCs were co-cultured with the progerin-expressing cells (Fig. 1d). In addition, CM from progerin-overexpressing MSCs induced senescence in non-senescent hMSCs, as determined by enhanced SA- $\beta$-gal activity (Fig. 1e). These results imply that progerin regulates paracrine transmission of senescence, which is referred to as SASP. The wellknown characteristics of SASP are an induction of senescence in adjacent cells and an attraction of inflammatory cells to eliminate the senescent cells ${ }^{20,31,32}$. To determine the ability of the progerin-overexpressing cells to attract inflammatory cells, we conducted cell migration assays using hUCB-derived MNCs. Compared to CM from control MSCs, CM collected from progerinexpressing cells caused increased migration in MNCs (Fig. 1f). These results suggest that the senescence phenotype induced by progerin in hMSCs can be transmitted into the neighboring cells by SASP factors.

\section{Progerin induces SASP via MCP-1 expression}

DNA damage associated with senescence induces cytokine secretion ${ }^{22,33}$. Progerin also induced the DNA damage response with an increased number of $\gamma \mathrm{H} 2 \mathrm{AX}$ foci (Fig. 2a). Because we found that senescence could be induced in hMSCs by co-culturing them with progerinoverexpressing hMSCs, we hypothesized that this paracrine senescence could be attributed to the induction of SASP factors. We explored senescence-associated cytokines in control, wt-LMNA, and progerin-overexpressing hMSCs (Fig. 2b). Interestingly, except for MCP-1, most cytokines that have been reported to increase in senescent fibroblasts were not increased in senescent hMSCs expressing progerin. The secretion of MCP-1 was also increased in the $\mathrm{CM}$ from progerin-expressing cells (Fig. 2c). MCP-1 mediates senescence through its receptor, $\mathrm{C}-\mathrm{C}$ chemokine receptor type $2(\mathrm{CCR} 2)^{15,25}$. To confirm the roles of MCP-1 in the SASP triggered by the progerin, we cultured normal hMSCs with the CM from the indicated cells in combination with CCR2 inhibitor (Fig. 2d). Importantly, CCR2 inhibitor impaired the induction of senescence by $\mathrm{CM}$ from progerin-expressing hMSCs. Additionally, the overexpression of wt-LMNA did not exhibit paracrine senescence (Fig. 2c, d). DNA damage is a key regulator of $\mathrm{SASP}^{17,22,32}$, and progerin can induce DNA damage ${ }^{3,4,6}$. These results suggest that progerin induce MCP-1-mediated paracrine senescence due to the increased DNA damage response.

\section{GATA4 regulates SASP caused by progerin in hMSCs}

Because GATA4 is one of the regulators of SASP that induces senescence and depends on DNA damage, we assessed the abundance of GATA4 in progerin-expressing cells (Fig. 3a) ${ }^{24}$. We expressed control vector or GFPprogerin in the hMSCs from 3 donors and analyzed them 7 days later by western blot. Progerin significantly increased the expression of GATA4 in hMSCs. However, the increase in GATA4 was confirmed at the protein level, not at the mRNA level, as previously reported in irradiation-induced senescent fibroblasts (Supplementary Figure 1a). Kang and colleagues reported that GATA4 is activated in response to DNA damage and is regulated by ataxia telangiectasia mutated (ATM) and ataxia telangiectasia and Rad3-related (ATR) ${ }^{24}$. We also investigated whether the inhibition of ATM and ATR with caffeine treatments in progerin-expressing cells could decrease GATA4 expression. Treatment with caffeine suppressed GATA4 in the progerin-expressing hMSCs (Fig. 3b). The results suggest that the activation of GATA4 in progerin-induced senescence is regulated by a similar mechanism as in the senescent fibroblasts. 
a

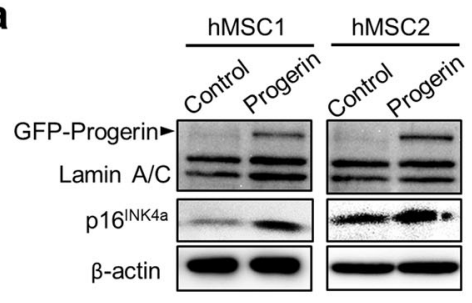

b



C

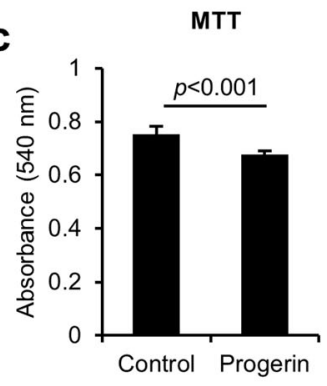

e

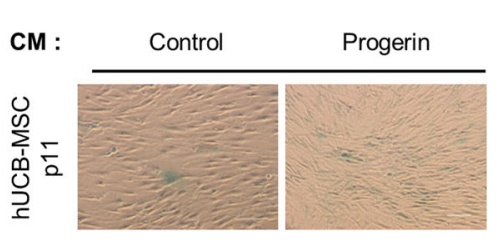

d
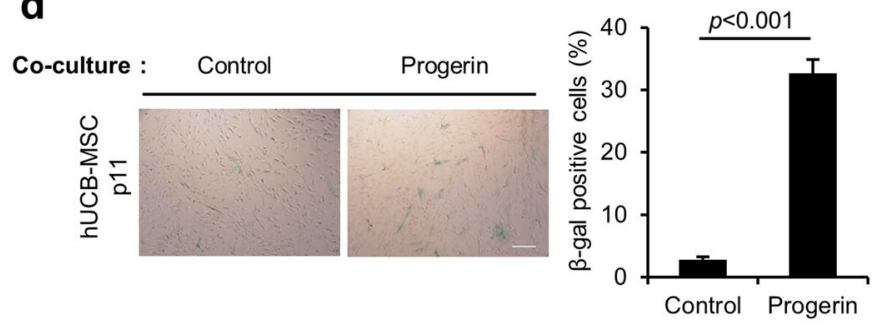

f

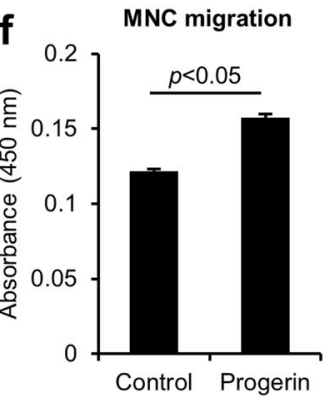

Fig. 1 Progerin overexpression triggers senescence in hMSCs, including secretory phenotypes. a-f hMSCs were infected with retroviruses encoding GFP-progerin to induce senescence or with the control vector at the same MOI. The cells were selected for 4 days with $0.5 \mu \mathrm{g} / \mathrm{ml}$ puromycin. After the selection, cells were cultured for 3 days before the analysis. a Immunoblotting analysis showing the overexpression of GFPprogerin protein and increased expression of $\mathrm{p} 16^{\mathrm{INK} 4 \mathrm{a}}$ in hMSCs. b (Left) Representative images of hMSCs stained for SA- $\beta$-gal after senescence induction by progerin. (Right) Quantification of percentage SA- $\beta$-gal-positive cells. Scale bar $=300 \mu \mathrm{m}$. c MTT assay to assess the proliferation of control and progerin-expressing MSCs $(n=3)$. $\mathbf{d}$, e (Left) Representative images of SA- $\beta$-gal staining of hMSCs. (Right) Quantification of percentage SA- $\beta$-gal-positive cells $(n=3)$. $\mathbf{d}$ hMSCs were co-cultured with control and progerin-overexpressing cells for 4 days in a Transwell chamber, and SA- $\beta$ gal activity was measured. Control and progerin-expressing cells were seeded in the upper chamber, and normal hMSCs were cultured in the lower chamber. The Transwell chamber blocks physical contact between upper- and lower-chamber cells. Scale bar $=100 \mu \mathrm{m}$. e hMSCs were cultured with CM from control and progerin-expressing hMSCs for 4 days, and SA- $\beta$-gal staining was performed. Scale bar $=200 \mu$ m. $\mathbf{f}$ Mononuclear cells isolated from human umbilical cord blood were placed in the top chamber, and CM from control or progerin-expressing MSCs was introduced in the lower chamber. Cell migration was evaluated following $6 \mathrm{~h}$ of exposure using CCK-8 $(n=3)$. Error bars represent means \pm s.d. from three separate experiments. Student's $t$-test was used for the statistical analysis

Interestingly, caffeine treatments in progerin-expressing hMSCs significantly decreased the mRNA expression level of MCP-1 (Fig. 3c). Caffeine treatment before the concentration of $\mathrm{CM}$ also abrogated senescence induction of CM from progerin-expressing hMSCs (Fig. 3d). Thus, we conclude that progerin-induced GATA4 expression is responsible for the MCP-1-mediated SASP of hMSCs. As lysosomal autophagy has been implicated in regulating GATA4 in senescent fibroblasts, we assessed the effects of autophagy on the GATA4 expression in the progerinexpressing $\mathrm{hMSCs}^{24}$. Control and progerin-expressing cells were treated with bafilomycin A1 (a lysosomal autophagy inhibitor) or rapamycin (macro-autophagy activator). The activation of macro-autophagy had no effect on GATA4 expression. However, the inhibition of lysosomal autophagy with bafilomycin A1 increased the expression of GATA4 in progerin-expressing cells (Fig. 3e). Control-MSCs did not express MCP-1 in response to the treatment with bafilomycin A1 despite a slight increase in GATA4 (Supplementary Figure 1b). These findings imply that the lysosomal autophagic pathway regulates GATA4 during progerin-induced senescence in hMSCs. These results are consistent with the previous findings in senescent fibroblasts induced by 

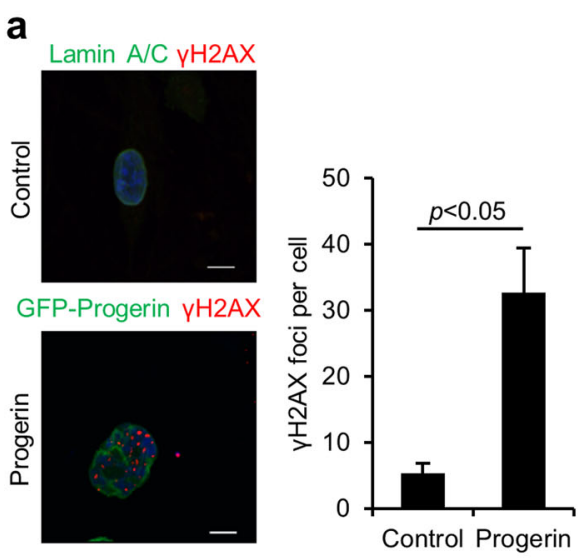

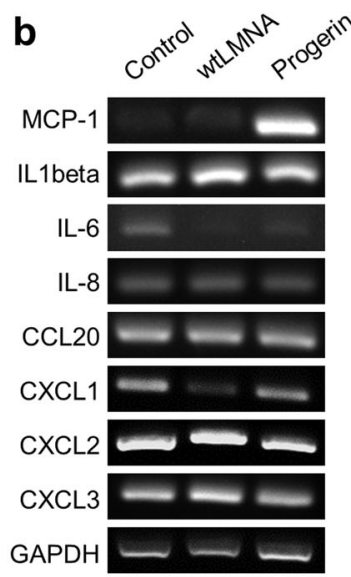

C

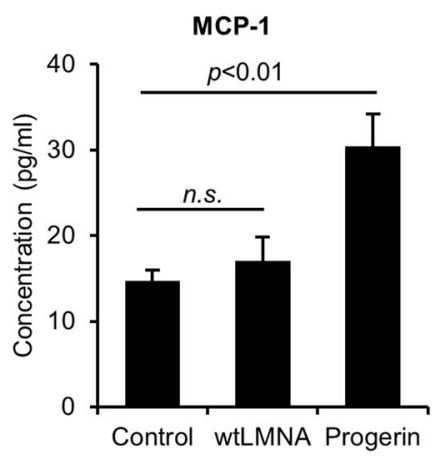

d

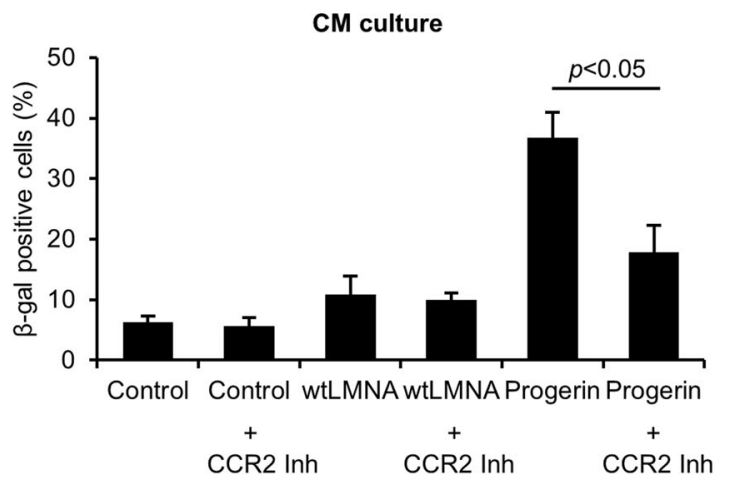

Fig. 2 Progerin-induced DNA damage signaling correlates with MCP-1 expression. a (Left) Representative images of immunofluorescence staining of $\mathrm{Y}-\mathrm{H} 2 \mathrm{AX}$-positive cells after transduction of GFP-progerin in hMSCs. (Right) Quantification of $\mathrm{y}-\mathrm{H} 2 \mathrm{AX}$ foci per cell $(n=30)$. Scale bar $=10$ $\mu \mathrm{m}$. b-d hMSCs were infected with retrovirus expressing GFP-wt-LMNA, GFP-progerin or empty vector, selected and cultured for 7 days. $\mathbf{b}$ PCR analysis was performed to evaluate cytokine expression in control, wt-LMNA, and progerin-expressing hMSCs. c CM was obtained from control, wtLMNA, and progerin-expressing hMSCs. The secretion level of MCP-1 was analyzed in the CM of each group using ELISA. d Non-senescent hMSCS were cultured with the indicated CM in the presence of CCR2 inhibitor (RS 102895, $100 \mathrm{nM}$ ) for 4 days. After the CM culture, SA- $\beta$-gal staining was performed to analyze the ratio of senescent cells. Error bars represent means \pm s.d. from three separate experiments. n.s.: not significant. Student's $t-$ test was used for the statistical analysis

irradiation. In the senescent fibroblast, GATA4 accumulates because of the decreased physical interaction of $\mathrm{p} 62$ and GATA4. To determine whether progerin also regulates the association of p62 and GATA4, we performed co-immunoprecipitation with GATA4 and GFP in control and GFP-progerin-expressing hMSCs. Importantly, the binding of GATA4 to p62 was confirmed in hMSCs, and the binding was decreased in response to the overexpression of progerin (Fig. 3f). The co-IP experiments revealed that GATA4 is bound to p62 for lysosomal degradation, and the binding is decreased in response to progerin. Because caffeine treatment decreased GATA4 and SASP of hMSCs, the decreased binding of p62 to GATA4 might have been due to the progerin-induced DNA damage, as in the previous reports using fibroblasts. These findings suggest that the DNA damage dependent expression of GATA4 in progerin-expressing hMSCs is responsible for the MCP-1-mediated SASP. Furthermore, the increased expression level of GATA4 is due to the decreased binding to p62 in progerin-expressing hMSCs.

\section{Depletion of GATA4 inhibits senescence in progerin- overexpressing hMSCs}

To determine whether GATA4 is necessary for the phenotype of progerin-induced senescence in hMSCs, we depleted GATA4 expression using retroviruses that expressed a short-hairpin RNA against GATA4 (shGATA4) in the progerin-expressing cells. Interestingly, the suppression of GATA4 in the progerin-expressing hMSCs significantly decreased SA- $\beta$-gal activity (Fig. 4a). However, the loss of GATA4 in the progerin-expressing hMSCs caused no change in the number of $\gamma-\mathrm{H} 2 \mathrm{AX}$ foci, suggesting that the inhibition of GATA4 could not 

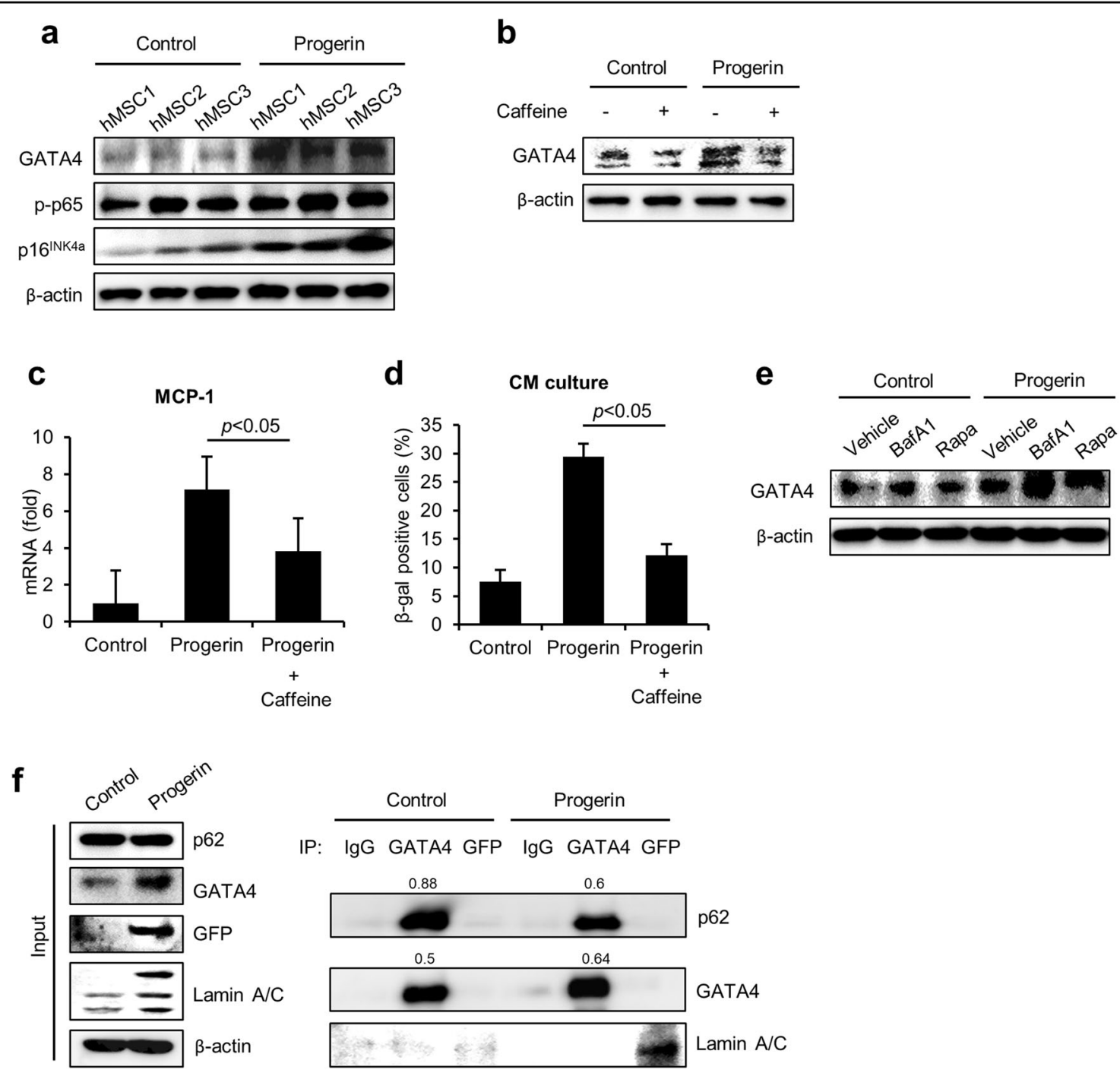

Fig. 3 GATA4 mediates the regulation of SASP induced by progerin. a After the introduction of GFP-progerin, GATA4 expression was detected using western blot analysis. $\mathbf{b}$ Western blot analysis of GATA4 was conducted in progerin-overexpressing MSCs after the treatment with caffeine (5 $\mathrm{mM}$ ) for $2 \mathrm{~h}$. c Quantitative PCR analysis of MCP-1 in progerin-overexpressing MSCs in the presence of caffeine (5 mM) for $2 \mathrm{~h}$. $\mathbf{d}$ hMSCs were cultured with CM from control or progerin-expressing hMSCs in the presence of caffeine. After 4 days of CM culture, the indicated cells were fixed and stained for the SA- $\beta$-gal activity. e Control and progerin-overexpressing hMSCs were treated with bafilomycin A1 (200 nM) or rapamycin (0.68 $\mu \mathrm{M})$ for $7 \mathrm{~h}$. The abundance of GATA4 protein was analyzed by western blotting. $\mathbf{I}$ Immunoblotting of GATA4 and GFP immunoprecipitates from control or progerinexpressing hMSCs. hMSCs were treated with bafilomycin A1 for $7 \mathrm{~h}$ before sampling to block GATA4 degradation. Error bars represent means \pm s.d. from three separate experiments. Student's $t$-test was used for the statistical analysis

protect the cells from progerin-induced DNA damage (Supplementary Figure 2). In addition, GATA4 did not affect the differentiation potential of progerin-expressing hMSCs (Supplementary Figure 3$)^{11}$. To investigate the regulatory role of GATA4 in the SASP induced by progerin in hMSCs, we conducted migration assays and cell culture with CM (Fig. 4b, c). Importantly, GATA4 depletion impaired the ability of the progerin-expressing hMSCs to attract immune cells in the migration assay (Fig. 4b). The decrease in GATA4 also inhibited the induction of senescence mediated by $\mathrm{CM}$ from progerin- expressing cells (Fig. 4c). Because MCP-1 is an important regulator of the SASP in progerin-induced senescence, we assessed whether the depletion of GATA4 decreased MCP-1 expression. Interestingly, GATA4 downregulation suppressed MCP-1 gene expression in progerinexpressing hMSCs, suggesting that GATA4 regulates the SASP of hMSCs through MCP-1 (Fig. 4d). The secretion of MCP-1 was also decreased in progerin-expressing cells in response to the downregulation of GATA4 (Fig. 4e). The GATA4 downregulation did not affect control cells, which showed low secretion of MCP-1. The results are 
a
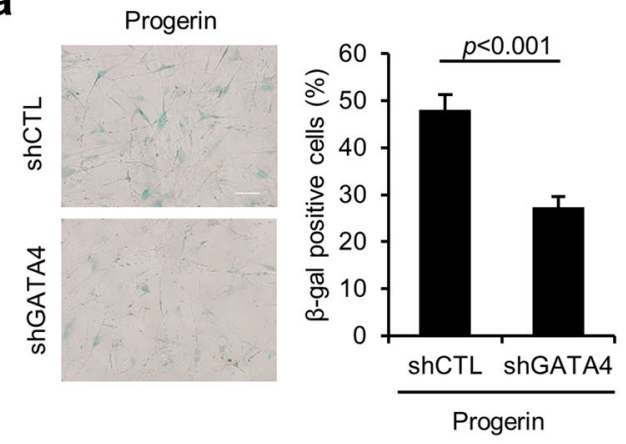

C

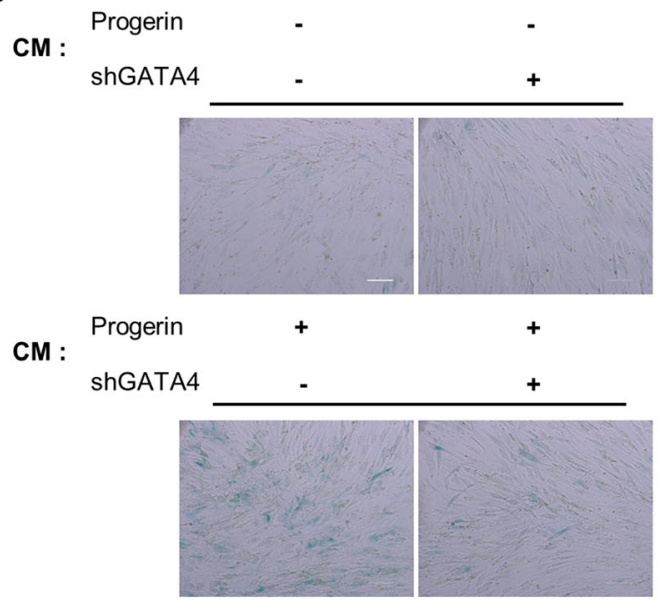

b

MNC migration



d

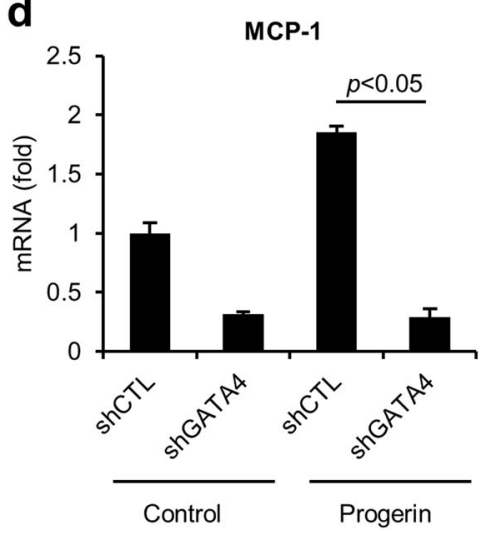

e

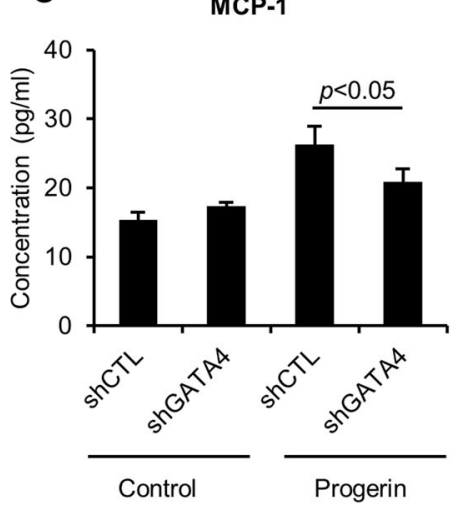

CM culture

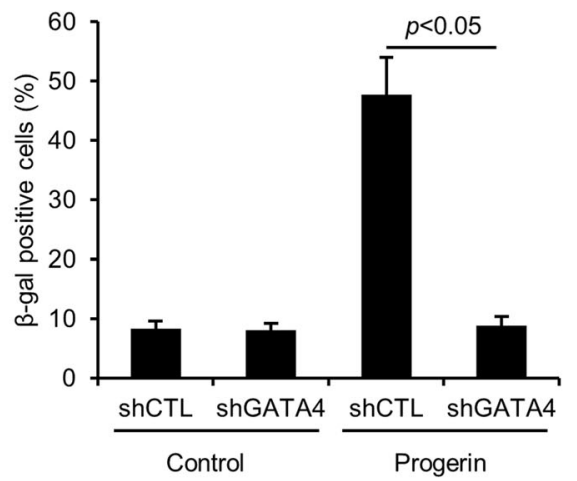

f

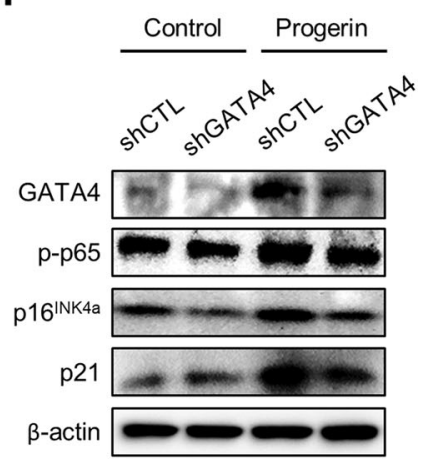

Fig. 4 Inhibition of GATA4 abrogates progerin-induced senescence in hMSCs. a-f Control and progerin-expressing hMSCs were infected with retroviruses encoding either control shRNA or GATA4-targeting shRNA. a SA- $\beta$-gal staining was determined using light microscopy (left) and counting (right) $(n=3)$. Scale bar $=200 \mu \mathrm{m}$. b CM was collected from shCTL- or shGATA4-infected progerin-expressing hMSCs. hUCB-MNCs were placed in the upper chamber, and CM was introduced into the lower chamber. Six hours later, cell migration was assessed using the Cell Counting Kit-8. c hUCB-MSCs were cultured for 4 days with the indicated CM, and induction of senescence was assessed with SA- $\beta$-gal staining. The graph shows the percentage of SA- $\beta$-gal-positive cells. Scale bar $=100 \mu \mathrm{m}$. $\mathbf{d}$ mRNA expression levels and e secretion levels of MCP-1 were measured in the indicated hMSCs. $\mathbf{f}$ The abundance of the indicated proteins was analyzed by western blotting. Error bars represent means \pm s.d. from three separate experiments. Student's $t$-test was used for the statistical analysis

consistent with the $\mathrm{CM}$ culture data (Fig. 4c). The GATA4-induced SASP is regulated by NF-kB activity ${ }^{24}$. The activation of NF- $\mathrm{kB}$ was also increased in response to progerin expression; however, the depletion of GATA4 decreased this activity (Fig. 4f). Together, these data show that knock-down of GATA4 abrogates progerin-mediated 

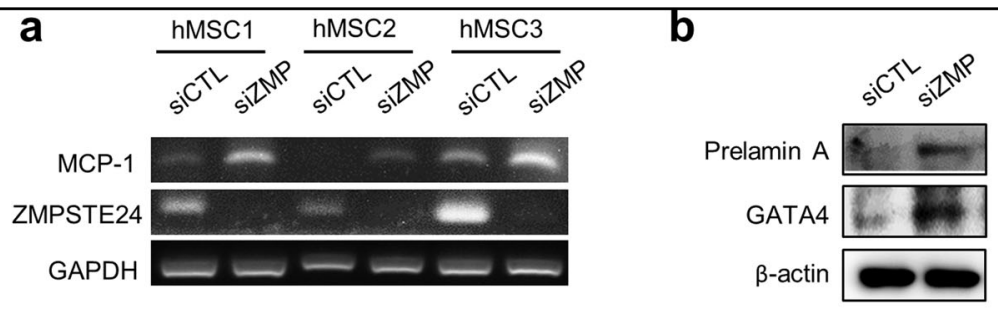

C
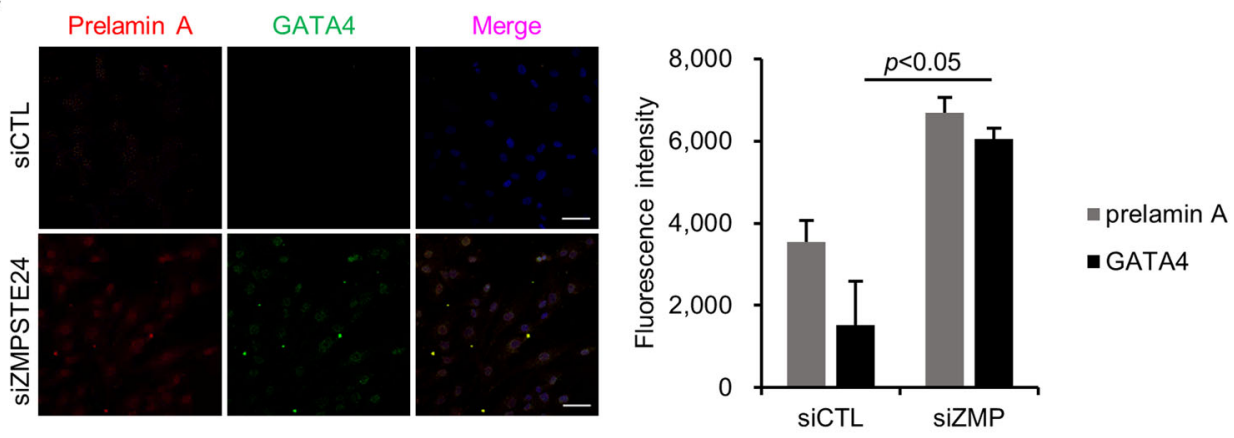

d


e
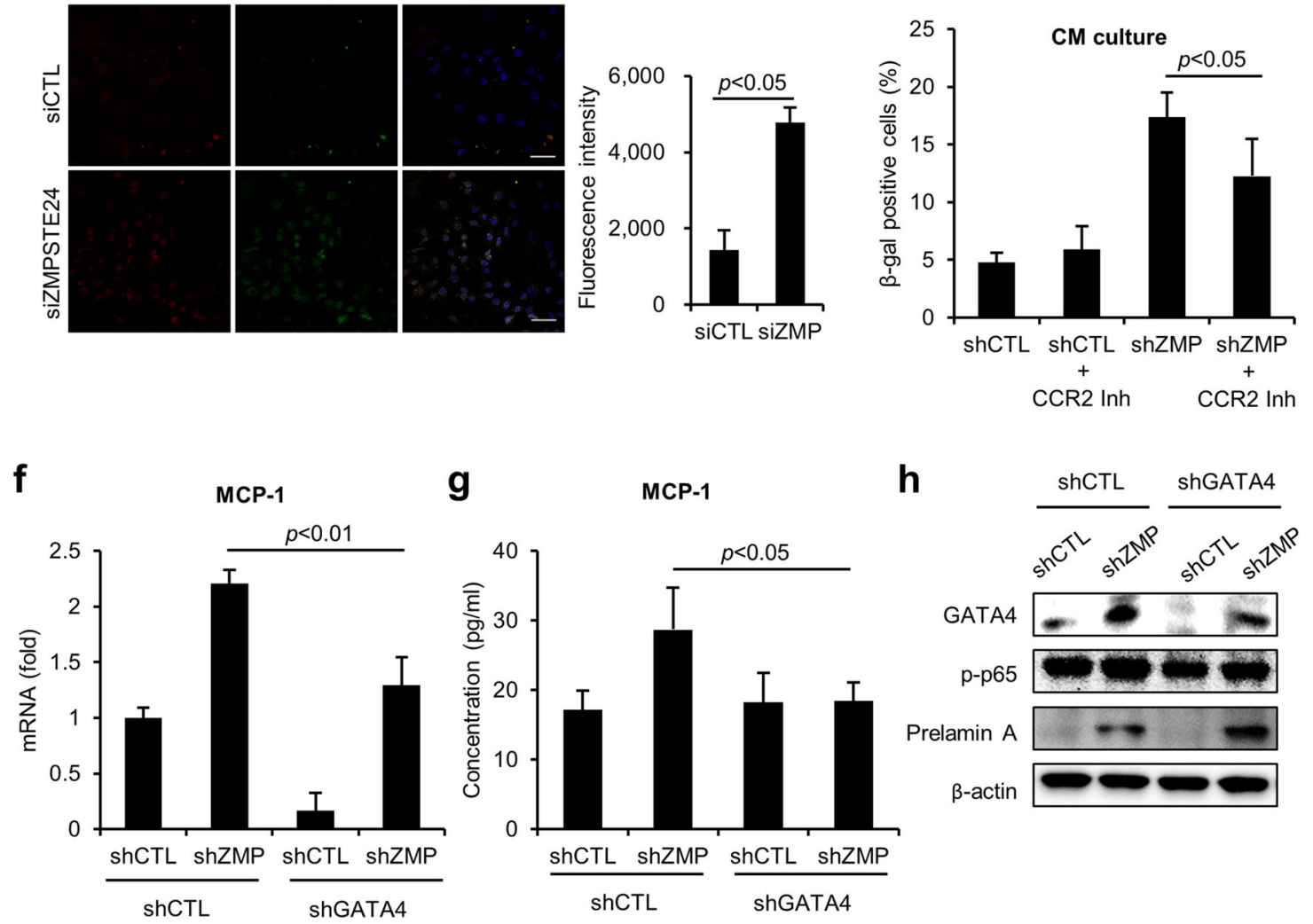

h

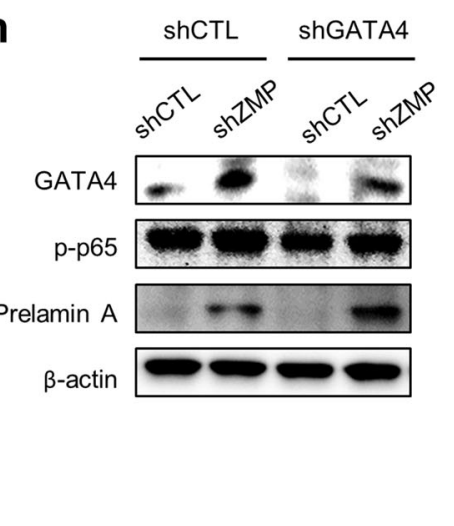

Fig. 5 GATA4 downregulation inhibits senescence in ZMPSTE24 depleted hMSCs. a After inhibition of ZMPSTE24 using siRNA, mRNA expression of MCP-1 and ZMPSTE24 was assessed by RT-PCR. b-d hMSCs were transfected with siControl and siZMPSTE24, and 2 days later, the indicated protein expression was determined. b Protein expression of prelamin A and GATA4 was analyzed by western blotting. c GATA4 and prelamin A expression in siCTL- and siZMPSTE24-transfected MSCs were determined by immunocytochemistry. Scale bar $=50 \mu \mathrm{m}$. $\mathbf{d}$ The expression of NF-KB was analyzed by immunofluorescence staining. Scale bar $=50 \mu \mathrm{m}$. e hMSCs were infected with retrovirus expressing either shControl or shZMPSTE24. CM was collected from ShCTL- and shZMPSTE24-infected hMSCs. hMSCs were grown in the CM in the presence of CCR2 inhibitor for 4 days. SA- $\beta$-gal staining was performed to determine senescent cells. $\mathbf{f}-\mathbf{h}$ hMSCs were infected with retrovirus expressing a shRNA targeting ZMPSTE24 in combination with retrovirus encoding a shRNA targeting GATA4. After co-transduction of shGATA4 and shZMPSTE24, $\mathbf{f}$ mRNA expression levels and $\mathbf{g}$ secretion levels of MCP-1 from the indicated cells were measured. $\mathbf{h}$ The indicated proteins were analyzed by western blotting. Error bars represent means \pm s.d. from three separate experiments. Student's $t$-test was used for the statistical analysis 


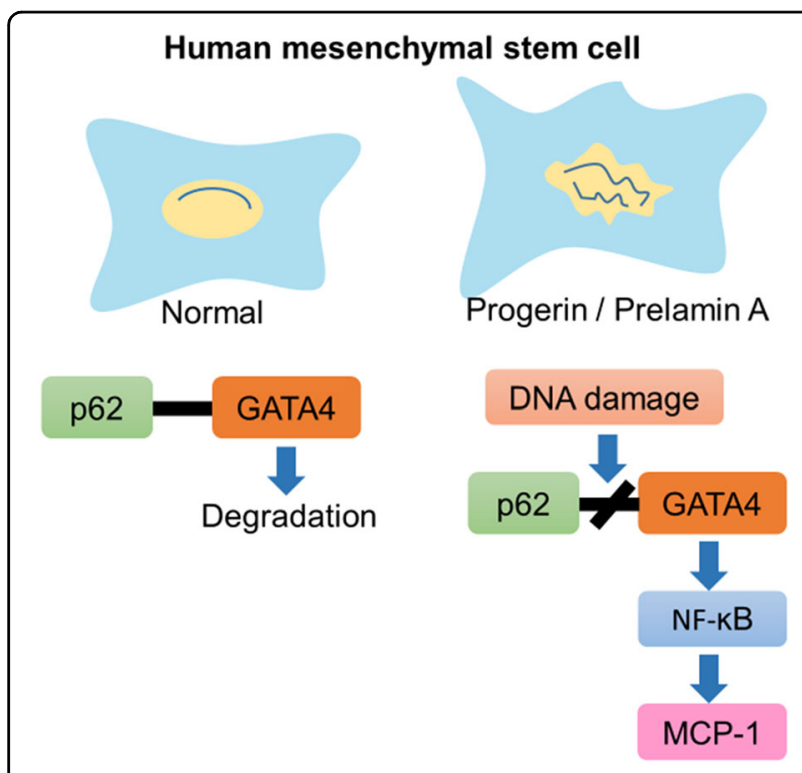

Fig. 6 Schematic representation of how GATA4 regulates senescence in $\mathrm{hMSC}$ s expressing progerin and prelamin $\mathrm{A}$. Progerin or prelamin A expression induces GATA4 expression through an increased DNA damage response and decreased binding to p62. The NF-kB pathway mediates GATA4-dependent MCP-1 expression

senescence induction and suppresses the SASP via the regulation of MCP-1.

\section{GATA4 downregulation decreases senescence induced by prelamin A}

ZMPSTE24 is a metalloproteinase responsible for the maturation of prelamin A, and the lack of ZMPSTE24 causes prelamin A accumulation. Mice deficient in Zmpste24 exhibit premature senescence resembling HGPS patients ${ }^{7,8}$. Moreover, according to our previous study, knock-down of ZMPSTE24 in hMSCs resulted in the accumulation of prelamin $\mathrm{A}$ and progeria-like abnormalities in the nucleus ${ }^{14}$. As we observed in progerin overexpression, the loss of ZMPSTE24 using siRNA induced MCP-1 expression (Fig. 5a). Importantly, GATA4 expression was increased in ZMPSTE24 knock-down cells (Fig. 5b, c). NF-kB activity was also increased in the ZMPSTE24-depleted hMSCs, suggesting that prelamin A triggers the inflammatory responses (Fig. $5 \mathrm{~d}$ ). To investigate whether prelamin A mediates the SASP of hMSCs, we knocked down ZMPSTE24 using shRNA. Normal hMSCs cultured with the CM of ZMPSTE24downregulated cells were induced to senescence, and the induction was decreased in the presence of CCR2 inhibitor (Fig. 5e). These results imply that the prelamin A-induced paracrine senescence is mediated by MCP-1 expression. Furthermore, caffeine treatment decreased GATA4, and bafilomycin A1 treatment induced GATA4 expression in ZMPSTE24 downregulated cells
(Supplementary Figure 4). These results suggest that the accumulation of GATA4 during ZMPSTE24 depletion is dependent on the DNA damage reaction and lysosomal degradation pathway. Next, we depleted GATA4 expression using shRNA and measured the expression of MCP-1 to assess the role of GATA4 in prelamin A-induced SASP. The knock-down of GATA4 inhibited the increase in MCP-1 in response to the loss of ZMPSTE24 (Fig. 5f, g). The downregulation of GATA4 also decreased the activity of NF-kB p65 consistent with the previous progerin models (Fig. $5 \mathrm{~h}$ ). These results suggest that GATA4 is also a mediator of SASP in ZMPSTE24-depleted senescent hMSCs. To assess the regulatory role of GATA4 in senescence of hMSCs, we expressed GATA4 using a Doxinducible (Tet-on) vector containing Flag-GATA4. GATA4 significantly increased the percentage of senescent cells, as shown by SA- $\beta$-gal staining (Supplementary Figure $5 \mathrm{a}$ ). The induction of senescence was also confirmed by increased expression of $\mathrm{p} 16^{\mathrm{INK} 4 \mathrm{a}}$ and $\mathrm{p} 21 \mathrm{using}$ western blot analysis (Supplementary Figure 5b). Importantly, GATA4 significantly increased the expression of MCP-1 mRNA (Supplementary Figure 5c). These findings imply that GATA4 plays a role in senescence of hMSCs and abnormal lamin A-induced MCP-1 expression (Fig. 6).

\section{Discussion}

Progerin or prelamin A induces cellular senescence and impairs the function of $\mathrm{MSCs}^{11-13}$. These studies have demonstrated that abnormal lamin A interferes with the differentiation potential of hMSCs and decreases the viability of cells transplanted in vivo. However, none of these studies have explored the secretory phenotype in senescent hMSCs with abnormal nuclei. Zmpste24 knock-out mice show increased expression of a series of SASP-associated cytokines in the liver through DNA damage-dependent NF- $\mathrm{kB}$ activation ${ }^{34}$. In addition, senescent vascular smooth muscle cells expressing prelamin A express multiple SASP factors and exhibit calcification ${ }^{35}$. We have previously reported that accumulation of progerin or prelamin A induces cellular senescence in hMSCs with an increase in DNA damage ${ }^{14}$. In this study, we assessed whether abnormal lamin A could regulate the secretory phenotype in hMSCs.

We found that overexpression of progerin and accumulation of prelamin A by ZMPSTE24 depletion led to senescence of neighboring hMSCs. More interestingly, among various cytokines and chemokines, only MCP-1 mRNA was strongly expressed in senescent hMSCs induced by abnormal lamin A. Jin et al. have reported that late-passage hMSCs secrete MCP-1 through a pathway involving activated reactive oxygen species ${ }^{25}$. In our study, however, the expression of MCP-1 in hMSCs with progerin overexpression was dependent on DDR (Fig. 2a). In 
addition, specific inhibition of MCP-1 with an inhibitor against its receptor, CCR2, abrogated paracrine senescence induction by progerin and prelamin A (Figs. 2d and 5e). A previous study using Zmpste24 knock-out mice and Lmna ${ }^{\mathrm{G} 609 \mathrm{G} / \mathrm{G} 609 \mathrm{G}}$ mice demonstrated that defects in nuclear lamin A trigger systemic inflammation, with increased serum levels of IL-6, CXCL1, and TNF- $\alpha^{34}$. In contrast, our results suggest that abnormal lamin A in hMSCs specifically increases MCP-1. These findings led us to focus on DNA damage and MCP-1 as an important axis of secretory phenotype in hMSCs with defective lamin A.

GATA4 regulates cellular senescence via DDR in fibroblasts ${ }^{24}$. However, it was unclear whether GATA4 could act as a regulator of senescence in other cell types and disease models. Interestingly, in our study, the accumulation of progerin or prelamin A increased GATA4 expression in hMSCs. In one previous study, DNA damage-induced GATA4 activated various SASP factors through increased NF-kB activity in fibroblasts ${ }^{24}$. However, in our study, progerin- or prelamin A-induced GATA4 specifically increased MCP-1 in hMSCs. We also confirmed the abundance of GATA4 was at the protein level but not at the mRNA level. Thus, we aimed to elucidate the role of progerin in the expression of GATA4. Because the expression of GATA4 was increased in response to the inhibition of lysosomal autophagy through the treatment with bafilomycin A1 in progerinexpressing hMSCs, we hypothesized that progerin could affect the lysosomal degradation of GATA4. Surprisingly, the binding of p62 to GATA4 was decreased in the progerin-expressing hMSCs. Thus, we concluded that lysosomal autophagy determines the expression of GATA4 via the interaction with the p62 in progerin model. More importantly, downregulation of GATA4 inhibited senescence induction by progerin or prelamin A in hMSCs. These results indicate that GATA4 plays a critical role in abnormal lamin A-mediated senescence in hMSCs. Furthermore, the role of GATA4 could be explored in other senescence pathways.

hMSCs exert immunosuppressive effects through the secretion of soluble factors ${ }^{29,36,37}$. Because of their potent therapeutic potential, hMSCs from diverse tissues have been used in an attempt to treat inflammatory diseases ${ }^{38,39}$. We have previously reported that acute and chronic inflammatory disorders can be rescued by antiinflammatory cytokines secreted by hMSCs ${ }^{29,40,41}$. However, it remained unclear whether the inflammatory cytokines expressed by senescent MSCs affect the therapeutic properties of these cells. Only a few studies have demonstrated the paracrine effects of senescent hMSCs in inducing senescence in neighboring $\mathrm{MSCs}^{25,26}$. Jin et al. demonstrated that knock-down of CCR2 improved the therapeutic efficacy of $\mathrm{hMSCs}^{25}$. Because we demonstrated that GATA4 mediates senescence and inflammation in this study, it is worth investigating whether GATA4 regulates the therapeutic efficacy of MSCs in various disease models. In addition, it would be interesting to investigate the interaction of factors involved in therapeutic effects and paracrine senescence in hMSCs.

In conclusion, this study revealed that abnormal nuclear membrane proteins may induce senescence in hMSCs in a paracrine manner. Furthermore, we identified GATA4 as a mediator of the SASP in hMSCs with defective lamin A and showed that the DNA damage response and defects in p62 binding for lysosomal degradation are involved in the expression of GATA4. Our findings provide new insights into the mechanism of lamin A-mediated senescence and senescence-associated inflammation.

\section{Acknowledgements \\ We are grateful to Stephen J. Elledge (Harvard Medical School, USA) for the GATA4 plasmids. We also thank Chanhee Kang (Seoul National University, Republic of Korea) for the critical advice and discussion. This work was performed with the support of the Cooperative Research Program for Agriculture Science \& Technology Development (Project No. PJ01100201), Rural Development Administration, Republic of Korea.}

\section{Author details}

${ }^{1}$ Adult Stem Cell Research Center, College of Veterinary Medicine, Seoul National University, Seoul 08826, Republic of Korea. ${ }^{2}$ College of Veterinary Medicine and Research Institute for Veterinary Science, Seoul National University, Seoul 08826, Republic of Korea. ${ }^{3}$ Hematology Branch, National Heart, Lung and Blood Institute, National Institutes of Health, Bethesda, MD 20892, USA. ${ }^{4}$ Pusan National University School of Medicine, Busan 49241, Republic of Korea. ${ }^{5}$ Biomedical Research Institute, Pusan National University Hospital, Busan 49241, Republic of Korea

\section{Authors' contributions}

J.Y.L. designed and performed the experiments and wrote the manuscript. K.-R.Y. designed the experiments and analyzed the data. B.-C.L., I.K., J.-J.K. and E.-J.J. performed the experiments. H.S.K., Y.S., and S.W.C. analyzed the data and reviewed the manuscript. K.-S.K. conceived and designed the study, analyzed the data, and prepared the manuscript.

\section{Conflict of interest}

The authors declare that they have no conflict of interest.

\section{Publisher's note}

Springer Nature remains neutral with regard to jurisdictional claims in published maps and institutional affiliations.

Supplementary information accompanies this paper at https://doi.org/ 10.1038/s12276-018-0092-3.

Received: 29 September 2017 Revised: 12 February 2018 Accepted: 8 March 2018.

Published online: 14 May 2018

\section{References}

1. Goldman, R. D. et al. Accumulation of mutant lamin A causes progressive changes in nuclear architecture in Hutchinson-Gilford progeria syndrome. Proc. Natl Acad. Sci. USA 101, 8963-8968 (2004).

2. Scaffidi, P. \& Misteli, T. Lamin A-dependent nuclear defects in human aging. Science 312, 1059-1063 (2006). 
3. Liu, B. et al. Genomic instability in laminopathy-based premature aging. Nat. Med. 11, 780-785 (2005)

4. Liu, Y., Rusinol, A., Sinensky, M., Wang, Y. \& Zou, Y. DNA damage responses in progeroid syndromes arise from defective maturation of prelamin A. J. Cell Sci. 119(Pt 22), 4644-4649 (2006).

5. Pendás, A. M. et al. Defective prelamin A processing and muscular and adipocyte alterations in Zmpste24 metalloproteinase-deficient mice. Nat. Genet. 31, 94-99 (2002).

6. Ugalde, A. P. et al. Aging and chronic DNA damage response activate a regulatory pathway involving miR-29 and p53. EMBO J. 30, 2219-2232 (2011)

7. Krishnan, V. et al. Histone $\mathrm{H} 4$ lysine 16 hypoacetylation is associated with defective DNA repair and premature senescence in Zmpste24-deficient mice. Proc. Natl Acad. Sci. USA 108, 12325-12330 (2011).

8. Liu, B. et al. Depleting the methyltransferase Suv39h1 improves DNA repair and extends lifespan in a progeria mouse model. Nat. Commun. 4, 1868 (2013).

9. Ghosh, S., Liu, B., Wang, Y., Hao, Q. \& Zhou, Z. Lamin A is an endogenous SIRT6 activator and promotes SIRT6-mediated DNA repair. Cell Rep. 13, 1396-1406 (2015).

10. Gonzalez-Suarez, I. et al. Novel roles for A-type lamins in telomere biology and the DNA damage response pathway. EMBO J. 28, 2414-2427 (2009).

11. Scaffidi, P. \& Misteli, T. Lamin A-dependent misregulation of adult stem cells associated with accelerated ageing. Nat. Cell Biol. 10, 452-459 (2008).

12. Liu, G. H. et al. Targeted gene correction of laminopathy-associated LMNA mutations in patient-specific iPSCs. Cell Stem Cell 8, 688-694 (2011).

13. Infante, A. et al. Prelamin A accumulation and stress conditions induce impaired Oct-1 activity and autophagy in prematurely aged human mesenchymal stem cell. Aging 6, 264-280 (2014).

14. $\mathrm{Yu}, \mathrm{K}$. R. et al. MicroRNA-141-3p plays a role in human mesenchymal stem cell aging by directly targeting ZMPSTE24. J. Cell Sci. 126(Pt 23), 5422-5431 (2013).

15. Acosta, J. C. et al. A complex secretory program orchestrated by the inflammasome controls paracrine senescence. Nat. Cell Biol. 15, 978-990 (2013).

16. Aird, K. M. et al. HMGB2 orchestrates the chromatin landscape of senescenceassociated secretory phenotype gene loci. J. Cell Biol. 215, 325-334 (2016).

17. Freund, A., Patil, C. K. \& Campisi, J. p38MAPK is a novel DNA damage responseindependent regulator of the senescence-associated secretory phenotype. EMBO J. 30, 1536-1548 (2011).

18. Herranz, N. et al. mTOR regulates MAPKAPK2 translation to control the senescence-associated secretory phenotype. Nat. Cell Biol. 17, 1205-1217 (2015).

19. Lasry, A. \& Ben-Neriah, Y. Senescence-associated inflammatory responses: aging and cancer perspectives. Trends Immunol. 36, 217-228 (2015).

20. Hoare, M. et al. NOTCH1 mediates a switch between two distinct secretomes during senescence. Nat. Cell Biol. 18, 979-992 (2016).

21. Laberge, R. M. et al. MTOR regulates the pro-tumorigenic senescence-associated secretory phenotype by promoting IL1A translation. Nat. Cell Biol. 17, 1049-1061 (2015).

22. Rodier, F. et al. Persistent DNA damage signalling triggers senescenceassociated inflammatory cytokine secretion. Nat. Cell Biol. 11, 973-979 (2009).

23. Kim, H. N. et al. DNA damage and senescence in osteoprogenitors expressing Osx1 may cause their decrease with age. Aging Cell 16, 693-703 (2017).
24. Kang, C. et al. The DNA damage response induces inflammation and senescence by inhibiting autophagy of GATA4. Science 349, aaa5612 (2015).

25. Jin, H. J. et al. Senescence-associated MCP-1 secretion is dependent on a decline in BMI1 in human mesenchymal stromal cells. Antioxid. Redox Signal. 24, 471-485 (2016).

26. Severino, V. et al. Insulin-like growth factor binding proteins 4 and 7 released by senescent cells promote premature senescence in mesenchymal stem cells. Cell Death Dis. 4, e911 (2013).

27. $Y u, K . R$. et al. A p38 MAPK-mediated alteration of COX-2/PGE2 regulates immunomodulatory properties in human mesenchymal stem cell aging. PLoS ONE 9, e102426 (2014).

28. Lee, J. Y. et al. BMl1 inhibits senescence and enhances the immunomodulatory properties of human mesenchymal stem cells via the direct suppression of MKP-1/DUSP1. Aging 8, 1670-1689 (2016).

29. Kim, H. S. et al. Human umbilical cord blood mesenchymal stem cells reduce colitis in mice by activating NOD2 signaling to COX2. Gastroenterology $\mathbf{1 4 5}$, 1392-1403.e1-8 (2013).

30. Yu, K.-R. et al. Rapid and efficient direct conversion of human adult somatic cells into neural stem cells by HMGA2/let-7b. Cell Rep. 10, 441-452 (2015).

31. $\mathrm{Xu}, \mathrm{M}$. et al. JAK inhibition alleviates the cellular senescence-associated secretory phenotype and frailty in old age. Proc. Natl Acad. Sci. USA 112, E6301-E6310 (2015).

32. $\mathrm{Yu}, \mathrm{Q}$. et al. DNA-damage-induced type I interferon promotes senescence and inhibits stem cell function. Cell Rep. 11, 785-797 (2015).

33. Tchkonia, T., Zhu, Y., van Deursen, J., Campisi, J. \& Kirkland, J. L. Cellular senescence and the senescent secretory phenotype: therapeutic opportunities. J. Clin. Invest. 123, 966-972 (2013).

34. Osorio, F. G. et al. Nuclear lamina defects cause ATM-dependent NF-kappaB activation and link accelerated aging to a systemic inflammatory response. Genes Dev. 26, 2311-2324 (2012).

35. Liu, Y., Drozdov, I., Shroff, R., Beltran, L. E. \& Shanahan, C. M. Prelamin A accelerates vascular calcification via activation of the DNA damage response and senescence-associated secretory phenotype in vascular smooth muscle cells. Circ. Res. 112, e99-e109 (2013).

36. Akiyama, K. et al. Mesenchymal-stem-cell-induced immunoregulation involves FAS-ligand-/FAS-mediated T cell apoptosis. Cell Stem Cell 10, 544-555 (2012).

37. Ren, G. et al. Mesenchymal stem cell-mediated immunosuppression occurs via concerted action of chemokines and nitric oxide. Cell Stem Cell 2, 141-150 (2008).

38. Le Blanc, K. et al. Mesenchymal stem cells for treatment of steroid-resistant, severe, acute graft-versus-host disease: a phase II study. Lancet $\mathbf{3 7 1}$, 1579-1586 (2008).

39. Kim, H. S. et al. Clinical trial of human umbilical cord blood-derived stem cells for the treatment of moderate-to-severe atopic dermatitis: phase I/lla studies. Stem Cells 35, 248-255 (2017).

40. Kim, H. S. et al. Human umbilical cord blood mesenchymal stem cell-derived PGE2 and TGF-beta1 alleviate atopic dermatitis by reducing mast cell degranulation. Stem Cells 33, 1254-1266 (2015).

41. Shin, T. H. et al. Human umbilical cord blood-stem cells direct macrophage polarization and block inflammasome activation to alleviate rheumatoid arthritis. Cell Death Dis. 7, e2524 (2016). 